\title{
Structural phase transitions in low-dimensional ion crystals
}

\author{
Shmuel Fishman, ${ }_{1}^{1}$ Gabriele De Chiara, ${ }^{2,3}$ Tommaso Calarco, ${ }^{4,5}$ and Giovanna Morigi ${ }^{2}$ \\ 1 Department of Physics, Technion, 32000 Haifa, Israel \\ 2 Grup d'Optica, Departament de Fisica, Universitat Autonoma de Barcelona, 08193 Bellaterra, Spain \\ 3 BEC-CNR-INFM \& Physics Department, University of Trento, Via Sommarive 14, I-38050 Povo (TN) Italy \\ 4 ITAMP, Harvard Smithsonian Center for Theoretical Atomic, \\ Molecular, and Optical Physics, Cambridge, MA, USA \\ 5 Abteilung Quanteninformationsverarbeitung, Universität Ulm, \\ Albert-Einstein-Allee 11, D-89069 Ulm, Germany
}

(Dated: November 11, 2018)

\begin{abstract}
A chain of singly-charged particles, confined by a harmonic potential, exhibits a sudden transition to a zigzag configuration when the radial potential reaches a critical value, depending on the particle number. This structural change is a phase transition of second order, whose order parameter is the crystal displacement from the chain axis. We study analytically the transition using Landau theory and find full agreement with numerical predictions by J. Schiffer [Phys. Rev. Lett. 70, 818 (1993)] and Piacente et al [Phys. Rev. B 69, 045324 (2004)]. Our theory allows us to determine analytically the system's behaviour at the transition point.
\end{abstract}

PACS numbers: 05.20.-y,52.27.Jt,61.50.-f

\section{INTRODUCTION}

Wigner crystals of ions in Paul or Penning traps are a remarkable example of selforganized matter at ultralow temperatures [1]. These systems are usually composed of singly-charged particles, which are kept together by external time-dependent radio-frequency or static magnetoelectric potentials, and which reach crystallization by means of laser cooling. Among several important aspects, the transition from disorder to order for few ions was studied in [2, 3, 4] ; long-range order in three dimensional structures in Penning traps was first demonstrated in [5, [6]; and more complex crystalline structures have been realized, see for instance [7, 8, 9]. Most recently, these crystalline structures have been attracting increasing attention for the realization of quantum information processors 10, 11, 12. and simulators 13, 14, 15, 16. In this perspective, the clear understanding and characterization of the structural properties would provide the possibility to control at the microscopic level the dynamics of complex systems. Moreover, ion crystals are systems characterized by truly long-range, unscreened Coulomb interactions, and hence constitute interesting physical systems where one can test equilibrium and out-of-equilibrium statistical mechanics models for systems exhibiting non-extensive thermodynamic functions [17, 18].

Structural transitions in ion crystals are induced either by changing the external potential [19, 20] or by introducing other forms of instabilities 21]. Structural transitions in low dimensional ion crystals were first characterized experimentally in [19, 20]. Here, starting from a chain configuration, the sudden transition to a planar zigzag structure, as shown in Fig. 1, was observed when the radial potential reached a critical value, dependent on the ion number. In theoretical investigations it was

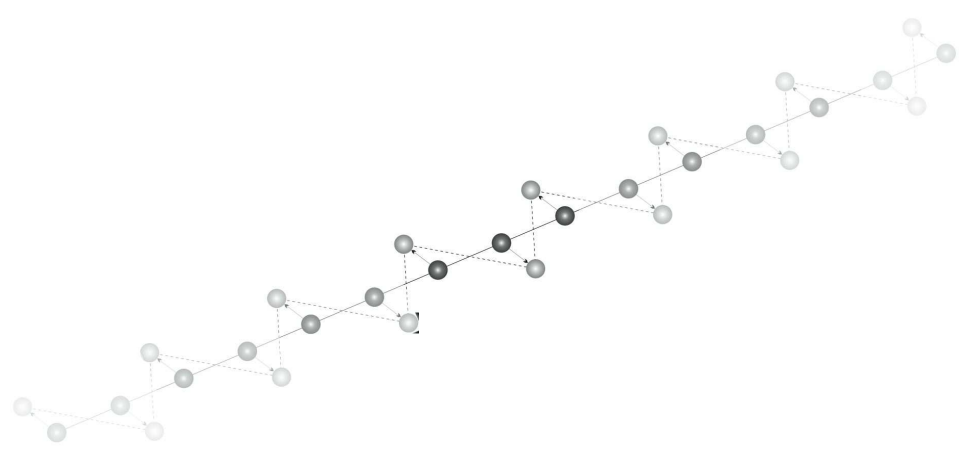

FIG. 1: Structural phase transition in a string of equidistant trapped ions from a linear to a planar zigzag configuration. For ions in a harmonic trap, close to the transition point the zigzag configuration is evident about the center of the trap, where the density of ion is larger [22].

conjectured that the structural change from a chain to a zigzag is a second order phase transition [22]. Further numerical work showed that at this transition point the ground state energy is characterized by a discontinuity in the second derivative with respect to the particles density [23].

In this article we study the structural phase transition of an ion crystal from a linear chain to a zigzag configuration in a suitably defined thermodynamic limit, by developing an analytic theory which allows us to determine the behavior of the system at the critical point. From symmetry considerations we conjecture the spontaneous symmetry breaking. Applying Landau theory 24], we identify the order parameter and the soft mode driving the instability, and demonstrate that the system undergoes a second order phase transition. Our theory is valid 
at $T=0$, when the system exhibits long-range order. It allows us to determine the system's behavior at the transition point, and the results we find are in agreement with the numerical results reported in [22] and in [23].

This article is organized as follows. In Sec. „ we introduce the model and discuss first the transition for a chain of 3 ions from a linear to a zigzag configuration of charges. In Sec. „ we derive the dispersion relations and eigenmodes at equilibrium of the linear chain and of the zigzag configuration in the thermodynamic limit. In Sec. we focus onto the classical phase transition between the two configurations, identify the soft mode and study analytically the system around the critical point. In Sec. we conclude and in the appendices we report the details of calculations presented in Sec. .

\section{ORDERED STRUCTURES OF IONS IN LOW DIMENSIONS}

The model we consider is constituted by $N$ particles of mass $m$ and charge $Q$, which are confined by an external harmonic potential along one axis. The particles are classical, and the Hamiltonian governing their dynamics reads

$$
H=\sum_{j=1}^{N} \frac{\mathbf{p}_{j}^{2}}{2 m}+V\left(\mathbf{r}_{1}, \ldots, \mathbf{r}_{N}\right)
$$

where $\mathbf{r}_{j}=\left(x_{j}, y_{j}, z_{j}\right)$ and $\mathbf{p}_{j}$ are the positions and conjugate momenta, with $j=1, \ldots, N$. The term $V$ accounts for the oscillator's potential and the Coulomb repulsion,

$V=\frac{1}{2} \sum_{j=1}^{N} m\left[\nu^{2} x_{j}^{2}+\nu_{t}^{2}\left(y_{j}^{2}+z_{j}^{2}\right)\right]+\frac{1}{2} \sum_{j=1}^{N} \sum_{j \neq i} \frac{Q^{2}}{\left|\mathbf{r}_{i}-\mathbf{r}_{j}\right|}$.

Here, the potential is characterized by harmonic confinement at frequency $\nu$ and $\nu_{t}$ in the axial and transverse direction, respectively, whereby $\nu_{t}>\nu$ for the case we are going to study. We denote by $\alpha \equiv \nu_{t} / \nu$ the trap aspect ratio, such that $\alpha>1$.

At sufficiently low temperatures, the ions localize themselves at the equilibrium positions $\mathbf{r}_{j}^{(0)}$ which solve the coupled equations describing the equilibrium of the forces,

$$
\left.\frac{\partial V}{\partial \mathbf{r}_{\mathbf{j}}}\right|_{\mathbf{r}_{\mathbf{j}}=\mathbf{r}_{\mathbf{j}}^{(\mathbf{o})}}=0 .
$$

When the transverse frequency $\nu_{t}$ exceeds a critical value $\nu_{t}^{(c)}$, which depends on the axial trap frequency $\nu$ and on the number of ions, the solutions of Eq. (3) are aligned along the $x$-axis, forming a string. Tables of the equilibrium positions for string up to 10 ions have been reported in 25, 26]. An analytical form for the linear density of ions along the trap axis at equilibrium was determined in [27] for $N \gg 1$ and using the local density approximation. The linear fluctuations about the classical ground state of an ion chain in a harmonic trap have been analytically studied in [28, 29]. This study identified as well the value of the critical transverse frequency $\nu_{t}^{(c)} \approx 3 N \nu /(4 \sqrt{\log N})$, using an expansion at leading order in $1 / \log N$, and by considering only nearest-neighbour contributions. Within this approximation this value is consistent with numerical results [22], and is in good agreement with previous analytical evaluations in [30], which calculated the critical value taking into account the long-range interaction between the ions but assuming that the particles are equidistant.

When the transverse frequency is varied, so that $\nu_{t}<$ $\nu_{t}^{(c)}$, the stable configuration is first a zigzag structure, then at smaller values it has an abrupt transition to a helicoidal one, and so on thereby acquiring more complex structures [19, 20, 30]. Eventually, for a large number of ions and for aspect ratios $\alpha$ sufficiently close to unity the structure is expected to take the b.c.c. crystalline form [1]. In the following, we study the transition from an ion chain to a zigzag structure for the most simple model, namely three ions in a linear Paul trap. This system allows us to get some insight into the system, before considering the structural transition in the thermodynamic limit in Sec. .

\section{Structural stability of a three-ion chain}

We consider $N=3$ ions inside a trap with $\nu_{t}>\nu$, and calculate their equilibrium positions as a function of the aspect ratio $\alpha=\nu_{t} / \nu$. We restrict for simplicity to two dimensions, which we here identify with the $x-y$ plane, and rewrite the potential (2) in dimensionless variables as

$$
\tilde{V}=\frac{1}{2} \sum_{i=1}^{3}\left({x^{\prime}}_{i}^{2}+\alpha^{2} y^{\prime 2}{ }_{i}\right)+\sum_{i<j} \frac{1}{\sqrt{\left(x_{i}^{\prime}-x_{j}^{\prime}\right)^{2}+\left(y_{i}^{\prime}-y_{j}^{\prime}\right)^{2}}},
$$

where $x_{i}^{\prime}=x_{i} / l, y_{i}^{\prime}=y_{i} / l, l^{3}=Q^{2} /\left(m \nu^{2}\right)$ and $\tilde{V}=$ $V /\left(l^{2} m \nu^{2}\right)$. Throughout this section we drop the prime superscript.

The normal modes frequencies for the linear and the zigzag structures are displayed in Fig. 2 as a function of $\alpha$, when $\alpha$ is decreased across the value for which the linear chain becomes mechanically unstable. The transition to the zigzag configuration takes place at the value $\alpha=\alpha^{*}$, such that the smaller transverse frequency of the linear chain vanishes. We now study in detail the classical equilibrium positions for $\alpha>1$. Assuming the convention $x_{1}<x_{2}<x_{3}$, the symmetry of the trapping potential imposes $x_{2}^{(0)}=0, x_{1}^{(0)}=-x_{3}^{(0)} \equiv-\bar{x}$, with $\bar{x}>0$. From Eqs. (3) we also find $y_{2}^{(0)}=-2 \bar{y}$ and 

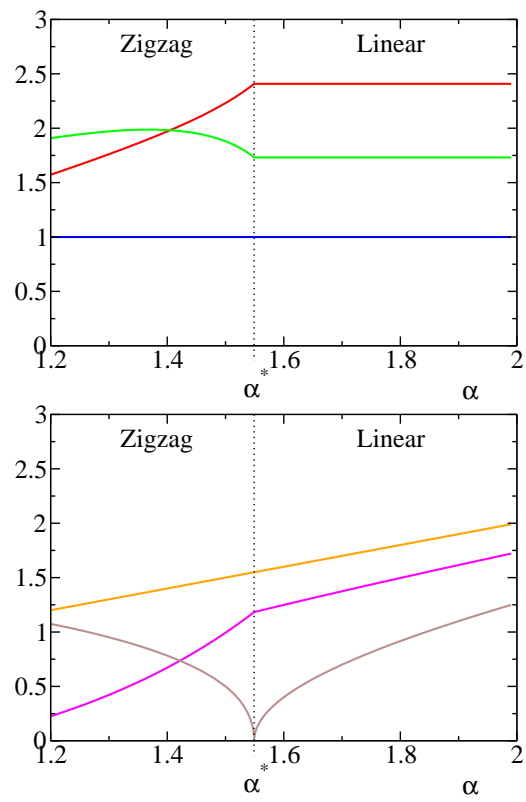

FIG. 2: (color online) Excitation frequencies of the oscillations modes of a three-ion string, in units of the axial trap frequency $\nu$, as a function of the trap anisotropy $\alpha$. The eigenmodes for $\alpha>\alpha^{*}$ are the longitunal (top) and the transverse ones (bottom). For $\alpha<\alpha^{*}$ the eigenmodes are combination of longitudinal and transverse modes with opposite parity by reflection about $x=0$.

$y_{1}^{(0)}=y_{3}^{(0)} \equiv \bar{y}$, with $\bar{y} \geq 0$. The linear configuration, namely the set of solutions with $\bar{y}=0$, is found when the aspect ratio $\alpha>\alpha^{*}$, where $\alpha^{*} \equiv \sqrt{12 / 5}$. When $\alpha<\alpha^{*}$, then $\bar{y}>0$ and the structure becomes planar. We denote this case by "zigzag configuration", as it is indeed the most elementary instance of the structure one observes for many ions. Here, for $\alpha<\alpha^{*}$ terms $\bar{x}, \bar{y}$ take the form

$$
\bar{x}=\left[4\left(1-\frac{\alpha^{2}}{3}\right)\right]^{-\frac{1}{3}}, \quad \bar{y}=\frac{1}{3} \sqrt{\left(\frac{3}{\alpha^{2}}\right)^{\frac{2}{3}}-\bar{x}^{2}},
$$

Their functional dependence on the aspect ratio $\alpha$ is displayed in Fig. 3. One can observe the discontinuity of the derivative at $\alpha^{*}$, corresponding to the transition to a different equilibrium configuration. For $\alpha \rightarrow \alpha^{*}$ the change is faster for the transverse displacement, as it is visible by the expansion of $\bar{x}, \bar{y}$ at $\delta \alpha=\alpha^{*}-\alpha$,

$$
\begin{aligned}
& \bar{y}=y_{0} \delta \alpha^{\frac{1}{2}}+\mathrm{O}\left(\delta \alpha^{3 / 2}\right) \\
& \bar{x}=\bar{x}_{\text {lin }}-x_{0} \delta \alpha+\mathrm{O}\left(\delta \alpha^{2}\right)
\end{aligned}
$$

where $\bar{x}_{\text {lin }}=(5 / 4)^{1 / 3}$ is the value taken by $\bar{x}$ when the linear chain is stable, while $y_{0} \approx 0.74, x_{0} \approx 1.85$. We note that, about the instability point of the linear chain, the transverse displacement $\bar{y}$ plays the role of the order parameter, while the changes of the axial distance $\bar{x}$ are induced by the changes of $\bar{y}$, and therefore about the value $\alpha^{*}$ these are less dramatic.

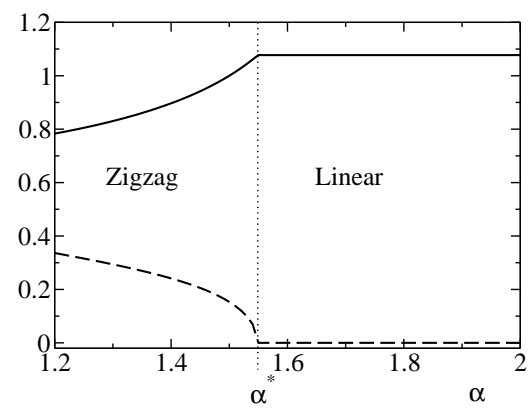

FIG. 3: Equilibrium position of the external ions of a string of 3 particles as a function of the trap anisotropy $\alpha$. The solid and dashed lines display the longitudinal and transverse variables, $\bar{x}$ and $\bar{y}$, in units of the characteristic length $l$. The vertical dotted line indicates the transition value $\alpha^{*}$, where the equilibrium configuration makes an abrupt change from a linear chain to a zigzag structure.

\section{THE LINEAR AND THE ZIGZAG STRUCTURES}

In this section, we study the static properties of the linear chain and of the zigzag configuration in the thermodynamic limit. For an ion chain inside a trap, a good thermodynamic limit is found by fixing the interparticle spacing $a$ at the chain center when $N \rightarrow \infty$. This corresponds to the requirement that the axial trap frequency vanishes according to the relation $\nu \sim \sqrt{\log N} / N[28,29]$. In this limit, the critical transverse frequency $\nu_{t}^{(c)}$ is constant, and the behaviour at the mechanical instability is equivalent to that of a uniform chain with equal interparticle distance $a$ between neighbouring ions 31. The uniform chain is the model we will use for determining the ground state and the motion of the linear and zigzag structure in the thermodynamic limit.

\section{The linear chain}

We assume a stable linear chain of ions, namely $\nu_{t}>$ $\nu_{t}^{(c)}$. In this limit the equilibrium positions lie along the $x$-axis, $\mathbf{r}_{j}^{(0)}=\left(x_{j}^{(0)}, 0,0\right)$, and we use the convention $x_{i}>$ $x_{j}$ for $i>j$. For small vibrations around these points we approximate the potential in Eq. (2) by its second order Taylor expansion in the displacements $q_{j}=x_{j}-x_{j}^{(0)}, y_{j}$, $z_{j}$. In this limit the equations of motion are

$$
\begin{aligned}
& \ddot{q}_{i}=-\nu^{2} q_{i}-\sum_{j \neq i} \frac{\mathcal{K}_{i, j}}{m}\left(q_{i}-q_{j}\right), \\
& \ddot{y}_{i}=-\nu_{t}^{2} y_{i}+\frac{1}{2} \sum_{j \neq i} \frac{\mathcal{K}_{i, j}}{m}\left(y_{i}-y_{j}\right), \\
& \ddot{z}_{i}=-\nu_{t}^{2} z_{i}+\frac{1}{2} \sum_{j \neq i} \frac{\mathcal{K}_{i, j}}{m}\left(z_{i}-z_{j}\right),
\end{aligned}
$$




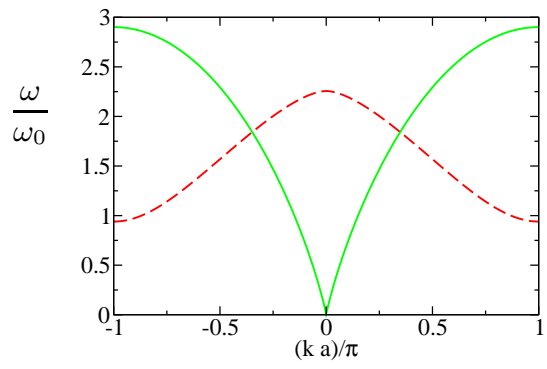

FIG. 4: (color online) Excitation spectrum of the uniform chain. The eigenfrequencies $\omega$, in units of $\omega_{0}=\sqrt{Q^{2} / m a^{3}}$, are plotted as a function of the quasimomentum $k$, in units of $\pi / a$. The axial spectrum (green solid line) and the transverse spectrum (red dashed line) are obtained from Eqs. (11) and (12), respectively. Here, $\nu_{t}=1.1 \nu_{t}^{(c)}$.

and describe a system of coupled oscillators, with long range interaction and position-dependent coupling strength. Here, the coefficients $\mathcal{K}_{i, j} \equiv-\partial^{2} V /\left.\partial x_{j} \partial x_{i}\right|_{x_{j}^{0}}$ read

$$
\mathcal{K}_{i, j}=\frac{2 Q^{2}}{\left|x_{i}^{(0)}-x_{j}^{(0)}\right|^{3}}
$$

We note that at second order in the harmonic expansion the axial and transverse vibrations are decoupled. It is easily verified that the center-of-mass motion is an eigenmode of the secular equations (6)-(8) at eigenfrequencies $\nu$ and $\nu_{t}$ for the axial and transverse motion, respectively. The solution to Eqs. (6)-(8) have been studied in [28, 29].

For the purpose of studying the behaviour at the mechanical instability, we now consider the simplified model of the uniform chain, where the interparticle distance at equilibrium is fixed. This case is found by setting $\nu=0$ in Eqs. (6)-(8) and assuming constant interparticle distance $a=x_{j+1}^{(0)}-x_{j}^{(0)}$. Such condition can be realized for the central ions of a long ion chain inside of a linear Paul trap [32] or for ions confined in a ring of large radius [19, 20]. This second scenario corresponds to take periodic boundary conditions, $q_{1}=q_{N+1}$, etc. Crystallization is found assuming, for instance, that one ion is pinned at the position $x_{0}^{(0)}=0$. Then, the classical equilibrium positions are $x_{j}^{(0)}=j a$ and the coupling strengths in Eqs. (6) - (8) take the form

$$
\mathcal{K}_{i, j}^{\text {uniform }}=\frac{2 Q^{2}}{|i-j|^{3} a^{3}} \equiv \mathcal{K}_{i-j}
$$

The dispersion relations are 33.

$$
\begin{array}{r}
\omega_{\|}(k)^{2}=4\left(\frac{2 Q^{2}}{m a^{3}}\right) \sum_{j=1}^{N} \frac{1}{j^{3}} \sin ^{2} \frac{j k a}{2}, \\
\omega_{\perp}(k)^{2}=\nu_{t}^{2}-2\left(\frac{2 Q^{2}}{m a^{3}}\right) \sum_{j=1}^{N} \frac{1}{j^{3}} \sin ^{2} \frac{j k a}{2},
\end{array}
$$

with $k=2 \pi n / N a$ and $n=0, \pm 1, \pm 2, \ldots, N / 2$. The spectrum corresponding to Eqs. (11) and (12) is shown in Fig. 4. The axial eigenmodes at frequency $\omega_{\|}(k)$ are $\Theta_{k}^{( \pm)}$, such that

$$
q_{j}=\sqrt{\frac{2}{N}} \sum_{k>0}\left(\Theta_{k}^{(+)} \cos k j a+\Theta_{k}^{(-)} \sin k j a\right),
$$

where the superscript \pm indicates parity by reflection $k \rightarrow-k$. Analogously, we denote the transverse eigenmodes at frequency $\omega_{\perp}(k)$ by $\Psi_{k}^{y( \pm)}$ and $\Psi_{k}^{z( \pm)}$, where

$$
\begin{aligned}
y_{j} & =\sqrt{\frac{2}{N}} \sum_{k>0}\left(\Psi_{k}^{y(+)} \cos k j a+\Psi_{k}^{y(-)} \sin k j a\right) \\
z_{j} & =\sqrt{\frac{2}{N}} \sum_{k>0}\left(\Psi_{k}^{z(+)} \cos k j a+\Psi_{k}^{z(-)} \sin k j a\right) .
\end{aligned}
$$

We note that the modes at $k=\pi / 2$ are even. A close inspection to Eq. (12) shows that there may exist values of the transverse trap frequency, at fixed interparticle distance $a$, for which $\omega_{\perp}^{2}<0$, that is, imaginary frequency solutions. For such values, thus, the chain is unstable. The threshold value $\nu_{t}^{(c)}$, such that for $\nu_{t}>\nu_{t}^{(c)}$ the linear chain is stable, is found by solving $\min _{k}\left(\omega_{\perp}\right)=0$ (see Sec. ${ }^{\text {) }}$. The minimum is found at $k=\pi / a$ and correspondingly

$$
\begin{aligned}
\nu_{t}^{(c) 2} & =2\left(\frac{2 Q^{2}}{m a^{3}}\right) \sum_{j=1}^{N} \frac{1}{j^{3}} \sin ^{2} \frac{j \pi}{2} \\
& \rightarrow \frac{Q^{2}}{m a^{3}} \frac{7}{2} \zeta(3),
\end{aligned}
$$

where result (16) is found for $N \rightarrow \infty$ using $\sum_{\ell>0}(2 \ell-$ $1)^{-p}=\left(1-2^{-p}\right) \zeta(p)$, with $\zeta(p)$ the Riemann-zeta function. The value in Eq. (16) depends on the interparticle spacing $a$ and provides the range of validity of the results presented in this section. It coincides with the value reported in [30], where a similar model to the one discussed here was considered. It is close to the result $\nu_{t}^{(c), \text { trap2 }}=4 Q^{2} / m a_{\text {trap }}(0)^{3}$ found at leading order in $1 / \log N$ in 29], where $a(x)$ gives the interparticle distance as a function of $x$ in the local density approximation, and $a(0) \equiv a$ is the value at the chain center. This result was obtained by considering the inhomogeneous distribution of ions along the chain, but keeping only the nearest-neighbours interaction. The small discrepancy between the two values is to be attributed to the different approximations that have been applied in each model.

\section{The zigzag structure}

For $\nu_{t}<\nu_{t}^{(c)}$, and sufficiently close to the critical value, the stable configuration is a zigzag structure. We now 


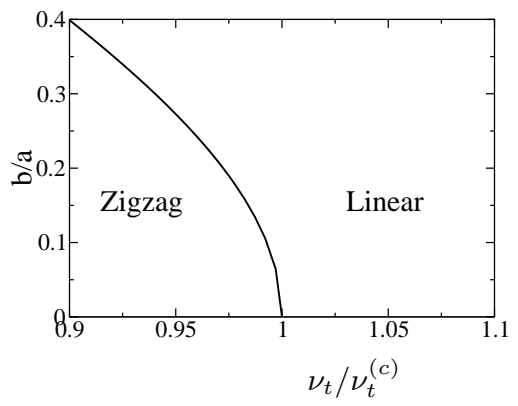

FIG. 5: Transverse equilibrium displacement $b$, in units of the interparticle spacing $a$, as a function of the transverse frequency $\nu_{t}$ in units of $\nu_{t}^{(c)}$. On the right of the curve the ion crystal is a linear chain. In the region on the left of the curve it exhibits a zigzag structure.

evaluate its dispersion relation and eigenmodes for ions on a ring and for periodic boundary conditions. We assume the equilibrium positions to lie on the $x-y$ plane with $\mathbf{r}_{n}^{(0)}=\left(x_{n}^{(0)}, y_{n}^{(0)}, 0\right)$. Then, $x_{n}^{(0)}=n a$ and $y_{n}^{(0)}=(-1)^{n} b / 2$, with $b$ a real and positive constant, which is determined from the equation

$$
\frac{m \nu_{t}^{2}}{Q^{2}}-\sum_{\ell>0} \frac{4}{\left[(2 \ell-1)^{2} a^{2}+b^{2}\right]^{3 / 2}}=0
$$

Figure 5 displays the transverse equilibrium displacement $b$ as a function of the transverse frequency $\nu_{t}$, as it is obtained by solving numerically Eq. (17). Assuming that the zigzag configuration is stable, we denote by $q_{n}=$ $x_{n}-x_{n}^{(0)}, w_{n}=y_{n}-y_{n}^{(0)}$, and $z_{n}$ the axial and transverse displacements, and expand the potential of Eq. (2) up to second order. In this limit the motion along the $z$ direction is decoupled from the vibrations on the plane, and the resulting equations of motion for $q_{n}$ and $w_{n}$ read

$$
\begin{aligned}
m \ddot{q}_{n}= & -\sum_{\ell \neq 0} \mathcal{K}_{\ell}^{x}\left(q_{n}-q_{n+\ell}\right) \\
& -(-1)^{n} \sum_{\ell \neq 0} \mathcal{Y}_{\ell}\left(w_{n}-w_{n+\ell}\right), \\
m \ddot{w}_{n}= & -m \nu_{t}^{2} w_{n}+\sum_{\ell \neq 0} \mathcal{K}_{\ell}^{y}\left(w_{n}-w_{n+\ell}\right) \\
& -(-1)^{n} \sum_{\ell \neq 0} \mathcal{Y}_{\ell}\left(q_{n}-q_{n+\ell}\right) .
\end{aligned}
$$

The coefficients appearing in these equations depend only on the interparticle distance, $\ell a=\left(n^{\prime}-n\right) a$, as the structure is periodic along $x$. In particular, for $\ell$ even they read

$$
\mathcal{K}_{\ell}^{x}=2 \mathcal{K}_{\ell}^{y}=\frac{2 Q^{2}}{a^{3}} \frac{1}{|\ell|^{3}}, \quad \mathcal{Y}_{\ell}=0,
$$

while for $\ell$ odd they are given by

$$
\begin{aligned}
& \mathcal{K}_{\ell}^{x}=\frac{Q^{2}}{a^{3}} \frac{2 \ell^{2}-\chi^{2}}{\left[\ell^{2}+\chi^{2}\right]^{5 / 2}}, \\
& \mathcal{K}_{\ell}^{y}=\frac{Q^{2}}{a^{3}} \frac{\ell^{2}-2 \chi^{2}}{\left[\ell^{2}+\chi^{2}\right]^{5 / 2}}, \\
& \mathcal{Y}_{\ell}=\frac{Q^{2}}{a^{3}} \frac{3 \ell \chi}{\left[\ell^{2}+\chi^{2}\right]^{5 / 2}},
\end{aligned}
$$

with $\chi=b / a$. The coefficients Eq. (10), and the corresponding equations of motion for the linear chain, Eqs. (6), (7), are recovered for $\chi \rightarrow 0$.

In general, the structural change brings to a doubling of the unit cell $d$ of the crystal, which from $d=a$ in the linear chain goes to $d=2 a$ in the zigzag configuration. Correspondingly, the Brillouin zone of the zigzag is reduced by a factor 2 , and the wave vectors now take the values $k=2 \pi n / N a$ and $n=0, \pm 1, \pm 2, \ldots, N / 4$. In Eqs. (18) and (19) one can easily verify that the bulk excitations are eigenmodes of the chain at frequencies $\nu$ and $\nu_{t}$. The other eigenvalues and eigenfunctions can be found using the ansatz $\mathbf{f}_{n}^{(j, \pm)}$, with

$$
\mathbf{f}_{n}^{(j, \pm)}(k)=( \pm 1)^{n} \mathrm{e}^{-i \omega_{j, \pm} t+i k n a}\left[\hat{\mathbf{x}} \mp i e^{-i n \pi} \epsilon_{k}^{(j, \pm)} \hat{\mathbf{y}}\right]
$$

where $j=1,2$ and $k a$ varies on the interval $[-\pi / 2, \pi / 2]$. In particular, we note the relation $\mathbf{f}_{n}^{(j,-)}(k)=\mathbf{f}_{n}^{(j,+)}(k+$ $\pi / a)$. The corresponding eigenmodes are given by the real and imaginary parts of these vectors. Using this ansatz, we obtain the coupled equations

$$
\begin{aligned}
& \omega_{j, \pm}(k)^{2}=C_{1}^{( \pm)}(k)+\epsilon_{k}^{(j, \pm)} B(k), \\
& \left(\nu_{t}^{2}-\omega_{j, \pm}(k)^{2}\right)=C_{2}^{( \pm)}(k)-\epsilon_{k}^{(j, \pm)^{-1}} B(k),
\end{aligned}
$$

whereby

$$
\begin{aligned}
& B(k)=\frac{2}{m} \sum_{\ell>0} \mathcal{Y}_{2 \ell-1} \sin (2 \ell-1) k a \\
& C_{1}^{(+)}(k)=\frac{4}{m} \sum_{\ell>0} \mathcal{K}_{\ell}^{x} \sin ^{2} \frac{k \ell a}{2} \\
& C_{2}^{(+)}(k)=\frac{4}{m} \sum_{\ell>0}\left(\mathcal{K}_{2 \ell}^{y} \sin ^{2} k \ell a+\mathcal{K}_{2 \ell-1}^{y} \cos ^{2} \frac{(2 \ell-1) k a}{2}\right), \\
& C_{1}^{(-)}(k)=\frac{4}{m} \sum_{\ell>0}\left(\mathcal{K}_{2 \ell}^{x} \sin ^{2} k \ell a+\mathcal{K}_{2 \ell-1}^{x} \cos ^{2} \frac{(2 \ell-1) k a}{2}\right), \\
& C_{2}^{(-)}(k)=\frac{4}{m} \sum_{\ell>0} \mathcal{K}_{\ell}^{y} \sin ^{2} \frac{k \ell a}{2}
\end{aligned}
$$

The eigenfrequencies are found by eliminating the parameter $\epsilon_{k}^{(j, \pm)}$ from Eqs. (21)-(22). The excitation spectrum exhibits four branches in the new Brillouin zone, and their functional dependence on $k$ is 


$$
\omega_{j, \pm}(k)^{2}=\frac{\nu_{t}^{2}+C_{1}^{( \pm)}(k)-C_{2}^{( \pm)}(k)}{2}+(-1)^{j} \sqrt{\frac{\left(\nu_{t}^{2}-C_{1}^{( \pm)}(k)-C_{2}^{( \pm)}(k)\right)^{2}}{4}+B(k)^{2}},
$$

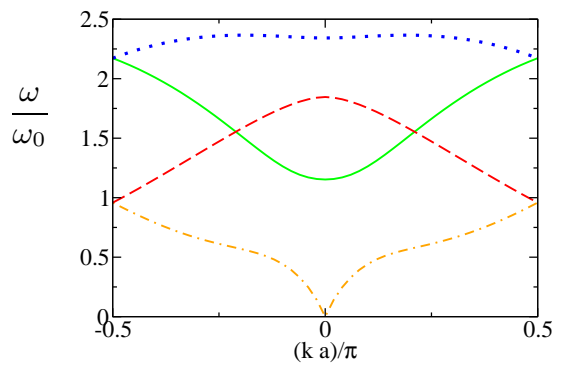

FIG. 6: (color online) Branches of the excitation spectrum of a zigzag structure for the modes on the $x-y$ plane, as obtained from Eq. (23). The curves display the frequencies $\omega_{2,+}(k)$ (green solid), $\omega_{2,-}(k)$ (blue dotted), $\omega_{1,+}(k)$ (orange dot-dashed), $\omega_{1,-}(k)$ (red dashed), in units of $\omega_{0}$, as a function of $k$, in units of $\pi / a$. The Brillouin zone is now half the Brillouin zone of the linear chain due to the doubling of the crystal periodicity. Here, $\nu_{t}=0.9 \nu_{t}^{(c)}$.

with $j=1,2$. The spectrum for the excitations on the $x-y$ plane is displayed in Fig. 6 .

We note that in the limit $b \rightarrow 0$ the branches of the spectrum of the linear chain, Eqs. (11) and (12), are recovered from Eqs. (23). In fact, for $b=0$ we have $B=0$ and $C_{1}^{( \pm)}=2 C_{2}^{(\mp)}$, such that each solution has double degeneracy, with

$$
\left.\omega_{2,+}(k)^{2}\right|_{b=0}=\left.\omega_{2,-}(k)^{2}\right|_{b=0}=\left.C_{1}^{(+)}(k)\right|_{b=0}
$$

and

$$
\omega_{1,+}(k)^{2}=\omega_{1,-}(k)^{2}=\nu_{t}^{2}-\left.C_{1}^{(+)}(k)\right|_{b=0} / 2,
$$

which reproduce respectively Eqs. (11) and (12) (note that $\left.\nu_{t}^{2}-C_{1}^{( \pm)}(k)-C_{2}^{( \pm)}(k)<0\right)$.

\section{LANDAU THEORY OF THE STRUCTURAL PHASE TRANSITION}

If the ions are crystallized along a line, by lowering the transverse confinement $\nu_{t}$ the system will be led to a situation in which the linear chain gets unstable. In this regime, one observes experimentally a transition, in which the ions are crystallized on a plane, according to a zigzag distribution of particles. In the literature it was conjectured that this is a second-order phase transition. This conjecture is supported by the numerical results in 22,23$]$.
Indeed, one can observe that the transition from a linear to a zigzag configuration is characterized by a symmetry breaking resulting in the increase of the unit cell by a factor of 2 . It is combined with a transition from a linear to a planar structure corresponding to the loss of rotational symmetry about the $x$-axis. Then, one can identify the order parameter with the displacement of the equilibrium position from the $x$-axis, while the control parameter can be taken as the transverse frequency $\nu_{t}$ when the interparticle distance is fixed. Starting from this educated guess we apply Landau theory to the transition 24]. We focus on the situation in which the interparticle distance $a$ is fixed, and study the crystal structure when the transverse confinement $\nu_{t}$ varies across the critical value $\nu_{t}^{(c)}$. We explicitly determine the critical exponent of the order parameter around the critical value, and find that it is in agreement with the numerical results in [22].

\section{The soft mode}

Let us now go back to the dispersion relation for the transverse modes of the linear chain in Eq. (12). The structural transition takes place for the critical value $\nu_{t}^{(c)}$, Eq. (16), such that the frequency of the lowest transverse mode of the linear chain vanishes, as shown in Fig. $7 \mathrm{a}$. The smallest transverse frequency $\omega_{\perp}$ is found at the value of the wave vector $k$, at which the semipositivedefinite function

$$
F(\varphi)=\sum_{j=1}^{N} \frac{1}{j^{3}} \sin ^{2}(j \varphi)
$$

is maximum in the interval $0 \leq \varphi \leq \pi / 2$, as seen from Eq. (12) for $k a=[0, \pi]$. We first observe that $\partial F / \partial \varphi=0$ at $\varphi=0, \pi / 2$. As $F(0)=0$, at $\varphi=0$ the function has an absolute minimum. The second-order derivative at $\varphi=$ $\pi / 2$ is negative, and one can simply prove analytically that this point is at least a relative maximum. Numerical studies show that it is an absolute maximum, such that the smallest transverse frequency is found at wave vector $k_{0} \equiv \pi / a$ and takes the value

$$
\omega_{\perp, \min }^{2}=\nu_{t}^{2}-\nu_{t}^{(c) 2} .
$$

This identifies the soft mode. The corresponding eigenmodes exhibit a periodic deformation of the chain at periodicity $2 a$, analogous to the zigzag structure. We denote 


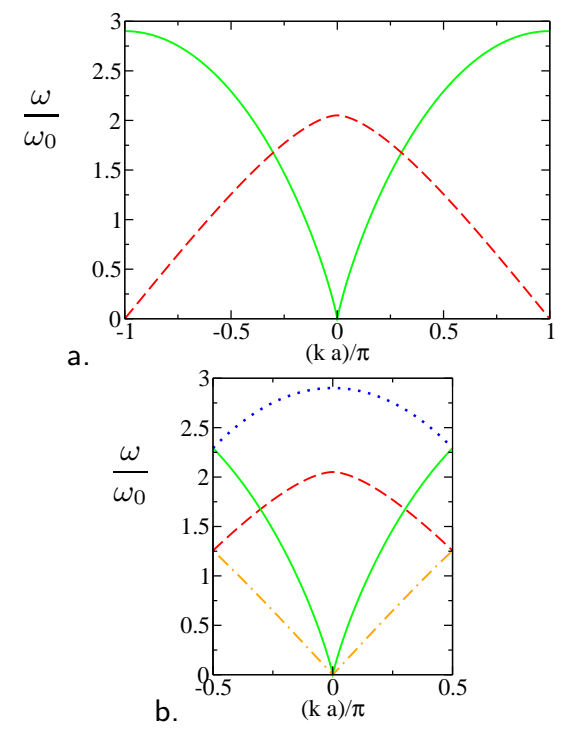

FIG. 7: (color online) Branches of the excitation spectrum at (a.) $\nu_{t}=\nu_{t}^{c}+0^{+}$(just above the critical value) and (b.) $\nu_{t}=\nu_{t}^{c}+0^{-}$(just below the critical value). In (b.) the equilibrium structure is a zigzag, the periodicity is doubled with respect to the linear chain and the new Brillouin zone is halfed. The four branches of the spectrum are obtained at this point by "folding" the two branches of the linear chain in (a.). The units and style codings in (a.) and (b.) are the same as in Figs. 4 and 6] respectively. Here, $\nu_{t}^{(c)} \simeq 2.05 \omega_{0}$.

by $b_{0}$ the amplitude of its oscillations, with $b_{0}=b_{0}\left(\nu_{t}\right)$, such that the transverse oscillations along $y$ of the ion $j$ are described by the function

$$
y_{j}^{\text {soft }}=(-1)^{j} b_{0} / 2 .
$$

In the following we assume zero temperature and study the equilibrium position of the crystal with the transverse frequency varying in the interval $\left[\nu_{t}^{(c)}-\delta \nu, \nu_{t}^{(c)}+\delta \nu\right]$, thus on both sides of the critical point, whereby $\delta \nu$ is a small positive quantity. Following Landau theory, we demonstrate that the zigzag mode of the linear chain, given by Eq. (25), is indeed the soft mode, driving the instability across the critical point, and we evaluate the critical exponents for some quantities of interest.

\section{Equilibrium positions around the critical point}

In order to determine the behaviour at the critical point, we first expand Eq. (2) till the fourth order around the equilibrium positions of the chain, $V=\sum_{l=1}^{4} V^{(l)}$, where $l$ labels the order. The zero order term at leading order in $1 / N$ is 27]

$$
V^{(0)}=\frac{Q^{2}}{a}(N-1)\left(\gamma-\ln 2+\ln (N)+\mathrm{O}\left(\frac{1}{N^{2}}\right)\right)
$$

where $\gamma=0.577216 \ldots$ is Euler's constant. The first order term vanishes as a result of the requirement that we are looking for a minimum. Using the decomposition into the eigenmodes of the linear chain, Eqs. (13)-(15), the quadratic term of the expansion of potential (2) (with the use of (6-9) and (11, 12) ) takes the form

$V^{(2)}=\frac{m}{2} \sum_{k>0, s= \pm}\left(\omega_{\|}(k)^{2} \Theta_{k}^{(s) 2}+\beta(k)\left(\Psi_{k}^{y(s) 2}+\Psi_{k}^{z(s) 2}\right)\right)$

where $\omega_{\|}$is given by Eq. (11), while

$$
\beta(k)=\nu_{t}^{2}-2\left(\frac{2 Q^{2}}{m a^{3}}\right) \sum_{j=1}^{N} \frac{1}{j^{3}} \sin ^{2} \frac{j k a}{2}
$$

and it coincides with $\omega_{\perp}^{2}(k)$, Eq. (12), for $\nu_{t}>\nu_{t}^{(c)}$. The third and fourth order terms, obtained by using this decomposition, are presented in App. .

The linear chain becomes mechanically unstable when, by varying $\nu_{t}$, the frequency of the mode with wave vector $k_{0}=\pi / a$, Eq. (25), vanishes. Starting from this observation, we study the behaviour of the corresponding mode close to the instability point, when $\nu_{t} \simeq \nu_{t}^{(c)}$. For convenience, we denote by $\Psi_{0}^{y}$ and $\Psi_{0}^{z}$ the zigzag modes of the linear chain along the $y$ and $z$ direction, respectively, at wave vector $k_{0}$. Around the instability point these modes will be coupled significantly to other quasidegenerate modes by the third and fourth order terms $V^{(3)}$ and $V^{(4)}$. These quasi-degenerate modes are long wavelengths axial modes $\Theta_{\delta k}$ at wave vectors $\delta k$, such that $|\delta k| a \ll 1$, and short wavelength transverse modes $\Psi_{k_{0}+\delta k^{\prime}}^{\sigma}$ at wave vector $k=k_{0}+\delta k^{\prime}$, with $\left|\delta k^{\prime}\right| a \ll 1$.

At first order in the small parameter $|\delta k| a \ll 1$, the part $V_{k_{0}}^{(3)}$, that contains the summands of the third order term $V^{(3)}$ giving the coupling of the mode at $k_{0}$ with the other quasi degenerate modes, has the form

$$
V_{k_{0}}^{(3)}=\frac{21}{2 \sqrt{2}} \zeta(3) \frac{Q^{2}}{a^{3} \sqrt{N}} \sum_{\delta k>0} \delta k \sum_{\sigma=y, z} \Psi_{0}^{\sigma}\left(\Theta_{\delta k}^{(+)} \Psi_{\delta k}^{\sigma(-)}+\Theta_{\delta k}^{(-)} \Psi_{\delta k}^{\sigma(+)}\right)+O\left(\delta k^{2} a^{2}\right),
$$

where we adopted for convenience the notation $\Psi_{\delta k}^{\sigma}:=\Psi_{k=k_{0}+\delta k}^{\sigma}$. We note that Eq. (29) is of first order in $\delta k$. The 
part $V_{k_{0}}^{(4)}$ of the fourth order term $V^{(4)}$, which is relevant to the dynamics of the soft mode at $k_{0}$, involves only the transverse modes that are close in $k$ to $k_{0}$, and has the form

$$
\begin{aligned}
V_{k_{0}}^{(4)}= & A\left(\Psi_{0}^{y 2}+\Psi_{0}^{z 2}\right)^{2}+12 A \sum_{\sigma=y, z} \Psi_{0}^{\sigma 2} \sum_{\delta k>0}\left[\Psi_{\delta k}^{\sigma(+)^{2}}+\Psi_{\delta k}^{\sigma(-)^{2}}\right]+4 A \sum_{\sigma=y, z ; \sigma^{\prime} \neq \sigma} \Psi_{0}^{\sigma 2} \sum_{\delta k>0}\left[\Psi_{\delta k}^{\sigma^{\prime}(+)^{2}}+\Psi_{\delta k}^{\sigma^{\prime}(-)^{2}}\right] \\
& +16 A \Psi_{0}^{y} \Psi_{0}^{z} \sum_{\delta k>0}\left[\Psi_{\delta k}^{y(+)} \Psi_{\delta k}^{z(+)}+\Psi_{\delta k}^{y(-)} \Psi_{\delta k}^{z(-)}\right]+A\left(\Psi_{0}^{y}+\Psi_{0}^{z}\right) \mathcal{F}\left(\Psi_{\delta k_{1}}^{\sigma(+)}, \Psi_{\delta k_{2}}^{\sigma^{\prime}(-)}, \Psi_{\delta k_{1}+\delta k_{2}}^{\sigma^{\prime \prime}(-)}+\mathrm{O}\left(\delta k^{2} a^{2}\right),(30)\right.
\end{aligned}
$$

where $A$ is calculated from the coefficients of $V^{(4)}$ at $k_{j}=$ $k_{0}(j=1,2,3,4)$, see App. ฉ and it takes the form $A=$ $\mathcal{A} / N$ with

$$
\mathcal{A}=\frac{3}{2} \frac{31}{32} \zeta(5) \frac{Q^{2}}{a^{5}} .
$$

The function $\mathcal{F}$ in Eq. (30) contains a sum of products of three amplitudes $\Psi_{\delta k}^{\sigma( \pm)}$ for $\delta k \neq 0$, and it is of no importance for the following considerations. The numerical factors appearing in Eq. (30), multiplying each term of the sum, account for all possible permutations of the amplitudes $\Psi_{\delta k}^{\sigma}$ in each summand (see App. ^and Eq. (47) therein). The coupling between the transverse modes at $k_{0}$ and the axial modes does not appear explicitly in Eq. (30), as it scales with $(\delta k a)^{2} \ll 1$, and it is hence of higher order with respect to the coupling among the transverse modes. Since the third order term, Eq. (29), scales with $\delta k a$, at zeroth order in the expansion in $|\delta k| a$ and close to the instability, the effective potential describing the dynamics of the mode at $k_{0}$ is given by (see Eqs. (27) and (30))

$$
\begin{aligned}
& V_{\text {eff }}=\frac{m}{2} \beta_{0}\left[\Psi_{0}^{y 2}+\Psi_{0}^{z^{2}}\right] \\
& +\frac{m}{2} \sum_{\delta k>0} \beta_{\delta k} \sum_{\sigma=y, z}\left[\Psi_{\delta k}^{\sigma(+)^{2}}+\Psi_{\delta k}^{\sigma(-)^{2}}\right]+V_{0}^{(4)},
\end{aligned}
$$

where $\beta_{\delta k} \equiv \beta\left(k_{0}-\delta k\right)$. We now allow the transverse frequency $\nu_{t}$ to take values in the interval $\left[\nu_{t}^{(c)}-\delta \nu, \nu_{t}^{(c)}+\delta \nu\right]$, such that $\beta_{\delta k}$ may take on small but negative values. We first determine the amplitude of the zigzag mode $k_{0}$ and then show that in the vicinity of the frequency $\nu_{t}^{(c)}$ no other modes are stable. For this purpose for $\beta_{\delta k}<0$ we determine the corrections $\bar{\Psi}_{\delta k}^{y( \pm)}, \bar{\Psi}_{\delta k}^{z( \pm)}$ to the equilibrium positions of the linear chain using Eq. (32), assuming that these give rise to a small displacement $b$ with respect to the equilibrium interparticle distance $a$, $b \ll a$. In particular, following our hypothesis that close to the transition point the soft mode is unique, and it is the zigzag mode, we consider the set of solutions where $\bar{\Psi}_{\delta k}^{( \pm)}=0$ for $\delta k>0$, and introduce the Fourier amplitude of the displacement in the transverse plane $\bar{\varrho}=\sqrt{N} b / 2$, as indicated from Eq. (13), such that

$$
\bar{\varrho}=\sqrt{\left(\bar{\Psi}_{0}^{y}\right)^{2}+\left(\bar{\Psi}_{0}^{z}\right)^{2}} .
$$

From Eq. (32) one finds $\bar{\varrho}=0$ for $\beta_{0}>0$, while for $\beta_{0}<0$

$$
\bar{\varrho}=\left(-N \frac{m \beta_{0}}{4 \mathcal{A}}\right)^{1 / 2} \text {. }
$$

This is indeed a minimum if we ignore terms in $V_{\text {eff }}$ with non-zero $\delta k$. It will be shown in what follows that this minimum is stable with respect to addition of such terms.

We now demonstrate that Eq. (33) is actually the transverse displacement, giving the equilibrium transverse positions of the zigzag structure, by verifying that Eq. (33), together with $\bar{\Psi}_{\delta k}^{( \pm)}=0$ for $\delta k>0$, yields a stable solution. To check stability the matrix of the second derivatives of $V_{\text {eff }}$ with respect to the various variables should be calculated. The second derivative of $V_{\text {eff }}$, given in Eq. (32). with respect to $\bar{\varrho}$ is positive,

$$
\left.\frac{\partial^{2} V_{\mathrm{eff}}}{\partial \varrho^{2}}\right|_{\left\{\varrho, \Psi_{\delta k}\right\}=\{\bar{\varrho}, 0\}}=-2 m \beta_{0}>0 .
$$

In order to investigate the coupling of the soft mode with the modes with $\delta k \neq 0$, one can calculate the second derivatives of $V_{\text {eff }}$ with respect to $\Psi_{0}^{\sigma}$. We find

$$
\left.\frac{\partial^{2} V_{\mathrm{eff}}}{\partial \Psi_{0}^{\sigma} \partial \Psi_{\delta k}^{\sigma^{\prime}( \pm)}}\right|_{\left\{\varrho, \Psi_{\delta k}\right\}=\{\bar{\varrho}, 0\}}=0 .
$$

This result shows that the derivatives with respect to $\Psi_{0}^{\sigma}$ form a sub-block of the stability matrix that can be diagonalized separately. All its eigenvalues are found to be positive. The other second derivatives at these points read

$$
\begin{aligned}
\left.\frac{\partial^{2} V_{\text {eff }}}{\partial \Psi_{\delta k}^{z( \pm) 2}}\right|_{\left\{\varrho, \Psi_{\delta k}\right\}=\{\bar{\varrho}, 0\}} & =m \beta_{\delta k}+8 A \bar{\varrho}^{2}+16 A \bar{\Psi}\left(\xi^{2} 25,\right) \\
\left.\frac{\partial^{2} V_{\text {eff }}}{\partial \Psi_{\delta k}^{y( \pm) 2}}\right|_{\left\{\varrho, \Psi_{\delta k}\right\}=\{\bar{\varrho}, 0\}} & =m \beta_{\delta k}+8 A \bar{\varrho}^{2}+16 A \bar{\Psi}(\xi \beta 6), \\
\left.\frac{\partial^{2} V_{\text {eff }}}{\partial \Psi_{\delta k}^{y( \pm)} \partial \Psi_{\delta k}^{z(\mp)}}\right|_{\left\{\varrho, \Psi_{\delta k}\right\}=\{\bar{\varrho}, 0\}} & =0 \\
\left.\frac{\partial^{2} V_{\text {eff }}}{\partial \Psi_{\delta k}^{y( \pm)} \partial \Psi_{\delta k}^{z( \pm)}}\right|_{\left\{\varrho, \Psi_{\delta k}\right\}=\{\bar{\varrho}, 0\}} & =16 A \bar{\Psi}_{0}^{y} \bar{\Psi}_{0}^{z}
\end{aligned}
$$

where we have used that $0>\beta_{\delta k}>\beta_{0}$. This result shows that the modes $\Psi_{\delta k}^{y( \pm)}$ and $\Psi_{\delta k}^{z( \pm)}$ are coupled in pairs. All 
contributions resulting of differentiation of the function $\mathcal{F}$ in Eq. (30) vanish. The stability matrix splits into $2 \times 2$ blocks that can be diagonalized separately. Using Eq. (33) and $\beta_{0}<\beta_{\delta k}$ one finds that the eigenvalues of each block are $m\left(\beta_{\delta k}-2 \beta_{0}\right)$ and $m\left(\beta_{\delta k}-6 \beta_{0}\right)$, hence both positive. Therefore, a gap opens between the soft mode frequency and the frequency of the modes at $\delta k \neq 0$ in the vicinity of the transition point. Therefore, the instability is driven by the soft modes with wave vector $k_{0}$, determining the order of the zigzag phase [34, 35].

\section{Behaviour at the critical point}

From Eq. (32), using the results of the previous section we can now write the effective potential for the soft modes, which reads

$$
V^{\mathrm{soft}}=\mathcal{V}\left(\left(\Psi_{0}^{y}\right)^{2}+\left(\Psi_{0}^{z}\right)^{2}\right)+A\left(\left(\Psi_{0}^{y}\right)^{2}+\left(\Psi_{0}^{z}\right)^{2}\right)^{2}
$$

where $A$ is given by Eq. (31) and

$$
\mathcal{V}=\frac{m}{2} \beta_{0}=\frac{1}{2} m\left(\nu_{t}^{2}-\nu_{t}^{(c) 2}\right) .
$$

Here, we have used that $\beta_{0}=\omega_{\perp, \text { min }}^{2}$, which in turn is given by Eq. (24). Hence, for $\mathcal{V}>0$ the potential $V^{\text {soft }}$ has a single minimum with $\Psi_{0}^{\sigma}=0$, and the linear chain is the ground state structure, while for $\mathcal{V}<0$ the potential landscape has the characteristic form of a Mexican hat with degenerate zigzag ground states at different angles around the symmetry axis. Indeed, while the order parameter $\varrho$ is fixed by condition (33), the ratio $\bar{\Psi}_{0}^{y} / \bar{\Psi}_{0}^{z}$ is arbitrary. The system hence possesses "Goldstone modes" at zero frequency, which are a consequence of the symmetry by rotations around the trap axis.

The transverse displacement from the trap is given from Eq. (33) by using Eq. (25) and the relation $b / 2=$ $\bar{\varrho} / \sqrt{N}$, which links the displacement in real space with its Fourier decomposition. Hence, for $\nu_{t}<\nu_{t}^{(c)}$ the transverse displacement from the trap depends on $\nu_{t}$ as

$$
b=\bar{b} \sqrt{\nu_{t}^{(c)}-\nu_{t}},
$$

with $\bar{b}=\sqrt{2 m \nu_{t}^{(c)} / \mathcal{A}}$. This behaviour is in agreement with the numerical results in 22].

From Eq. (41) we evaluate the difference between the ground state energy of the linear and of the zigzag structure. Considering the energy per particle, from Eq. (39) we find

$$
\begin{aligned}
& \Delta E=\frac{V^{\mathrm{soft}}\left(\nu_{t} \rightarrow \nu_{t}^{(c)-}\right)-V^{\mathrm{soft}}\left(\nu_{t} \rightarrow \nu_{t}^{(c)+}\right)}{N} \\
& =-\frac{1}{2} m \mathcal{C} a^{2}\left(\nu_{t}-\nu_{t}^{(c)}\right)^{2}
\end{aligned}
$$

where $\mathcal{C}=112 \zeta(3) /[93 \zeta(5)]$, and whose second derivative with respect to $\nu_{t}$ is clearly discontinuous at the critical point. This result is consistent with the result presented in 23], where a discontinuity in the second derivative of the ground state energy with respect to the particles density was found.

\section{Discussion}

Using symmetry arguments we have demonstrated that the transition from a linear chain to a zigzag structure, in a system of anisotropically confined charges, is a secondorder phase transition, whose order parameter is the displacement from the trap axis. This theory has been developed in the thermodynamic limit, fixing the interparticle distance $a$ as the number of ions was let to infinity. In this limit, we found that the soft modes are the zigzag modes of the linear chain, whose periodicity is equal to twice the interparticle distance $a$. The instability is thus driven by these modes as the transverse potential is changed across the critical value $\nu_{t}^{(c)}$.

These considerations are strictly valid for $N \rightarrow \infty$, but can still be useful for finite systems, and in particular when the ions are confined in a trap, which provides also axial harmonic confinement. While detailed quantitative predictions can be only made by accurately evaluating the finite-size corrections, we can still make some reasonable conjectures, based on previous results in the literature and on our theory. Inside a harmonic trapping potential, the interparticle distance between the ions varies along the chain and it is minimal at the center. Numerical results, based on molecular dynamics simulations, showed that in this case the zigzag structure appears at the center of the chain where the density is highest [22]. Analytical studies found that the short wavelength modes are characterized by largest displacements at the chain center, while the ions at the edge almost do not move 29]. In this case, hence, we can still identify the zigzag mode of the ion chain with the soft mode. In the presence of axial confinement, however, both the transverse as well as the axial equilibrium points will change. In particular, when going to the zigzag structure the axial density of ions in the center will increase. Close to the transition point, one finds that the axial corrections to the linear chain positions are much smaller than the transverse displacements from the trap axis as this is a quantity that follows the order parameter. This is also confirmed by the analysis made for the simple case of three ions in Sec. where close to the critical value of the aspect ratio the transverse displacement varies faster than the axial one. Therefore, we expect that our theory will still provide reasonable predictions close to the critical point, also in presence of axial confinement. 


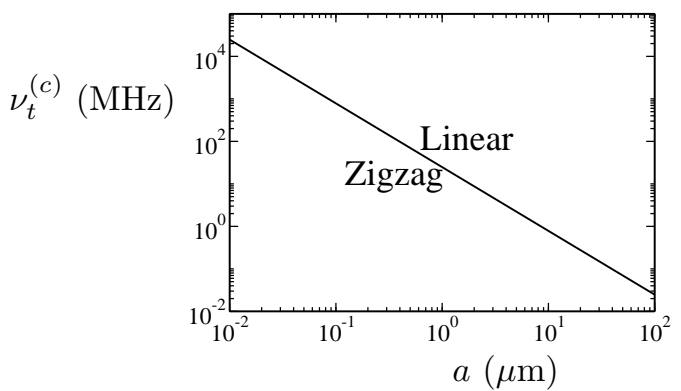

FIG. 8: Phase diagram close to the linear-zigzag transition in the thermodynamic limit, for ${ }^{40} \mathrm{Ca}^{+}$ions. The horizontal axis is the interparticle spacing $a$ in $\mu \mathrm{m}$, the vertical axis the corresponding critical frequency $\nu_{t}^{(c)}$ in MHz. This graphic does not report further curves in the left region, giving the transition to more complex structures. A detailed study of the transitions to these structures can be found in [19, 20] and [23].

\section{CONCLUSIONS AND OUTLOOK}

The structural phase transition from a linear chain to a zigzag configuration, in a system composed of trapped singly-charged particles, is a second order phase transition. Using a mean field approach we have derived a classical model, describing the system at the critical point and its vicinity. Our theory is analytical and its predictions agree with the numerical simulations of [22] and [23].

The corresponding phase diagram is shown in Fig. 8, it shows the regions of stability of the linear chain as a function of the interparticle spacing $a$ and the transverse frequency $\nu_{t}$. The phase diagram is evaluated in the thermodynamic limit, corresponding in keeping $a$ fixed as $N$ and the chain length go to infinity. The analysis is valid for $T=0$, where long-range order in one-dimensional structures exists. The quantum statistics of the particles at these densities seem irrelevant even at these ultralow temperatures since the interaction energy at all stages much larger than the kinetic energy, and the particles can be considered distinguishable at all effects [36]. On the other hand, at the critical point, where large fluctuations of the transverse motion classically occur, quantum effects may be relevant and could be in principle observed.

The authors thank Efrat Shimshoni, Grigory Astrakharchik, Eugene Demler, Bert Halperin, and Tommaso Roscilde for stimulating discussions and useful comments. This work was partly supported by the European Commission (EMALI, MRTN-CT-2006-035369; SCALA, Contract No. 015714; QOQIP, MOIF-CT-2005-8688), by the Spanish Ministerio de Educación y Ciencia (Consolider Ingenio 2010 "QOIT", CSD2006-00019; QLIQS, FIS2005-08257; Ramon-y-Cajal individual fellowship), and by the National Science Foundation through a grant to the Institute for Theoretical Atomic, Molecular, and
Optical Physics at the Smithsonian Center for Astrophysics and Harvard Department of Physics. S.F. acknowledges the US-Israel Binational Science Foundation (BSF), The Israel Science Foundation (ISF), the Minerva Center of Nonlinear Physics of Complex Systems, and the fund for Promotion of Research at the Technion. G.M. thanks Herbert Walther, who motivated this work.

\section{EXPANSION ABOUT THE EQUILIBRIUM POSISIONS OF THE LINEAR CHAIN}

In this Appendix we evaluate the higher order terms of the expansion of the potential in Eq. (2) about the equilibrium position of the linear chain. For this purpose we rewrite the interparticle distance as $\left|\mathbf{r}_{\mathbf{i}}-\mathbf{r}_{\mathbf{j}}\right|=$ $\sqrt{A_{i j}+\tau_{i j}+\epsilon_{i j}}$ with

$$
\begin{aligned}
& A_{i j}=(i-j)^{2} a^{2}, \\
& \tau_{i j}=2 a(i-j)\left(q_{i}-q_{j}\right), \\
& \epsilon_{i j}=\left(q_{i}-q_{j}\right)^{2}+\delta_{i j}^{2},
\end{aligned}
$$

and $\delta_{i j}^{2}=\left(y_{i}-y_{j}\right)^{2}+\left(z_{i}-z_{j}\right)^{2}$ and we have used $x_{j}=$ $j a+q_{j}$. We now expand in the parameters $\epsilon_{i j}$ and $\tau_{i j}$, assuming that they are small with respect to $A_{i j}$, i.e., to the axial equilibrium distances between the ions when the chain is stable. We will check later for consistency of this assumption. We can write

$$
V^{(l)}=\frac{Q^{2}}{2} \sum_{i, j \neq i} W_{i j}^{(l)}
$$

with

$$
\begin{aligned}
W_{i j}^{(0)} & =\frac{1}{|i-j| a}, \\
W_{i j}^{(1)} & =-\frac{\sigma_{i j}}{(i-j)^{2} a^{2}}\left(q_{i}-q_{j}\right), \\
W_{i j}^{(2)} & =\frac{1}{2|i-j|^{3} a^{3}}\left(2\left(q_{i}-q_{j}\right)^{2}-\delta_{i j}^{2}\right), \\
W_{i j}^{(3)} & =\frac{\sigma_{i j}}{2|i-j|^{4} a^{4}}\left(q_{i}-q_{j}\right)\left[3 \delta_{i j}^{2}-2\left(q_{i}-q_{j}\right)^{2}\right], \\
W_{i j}^{(4)} & =\frac{1}{|i-j|^{5} a^{5}}\left(\frac{3}{8} \delta_{i j}^{4}+\left(q_{i}-q_{j}\right)^{4}-3 \delta_{i j}^{2}\left(q_{i}-q_{j}\right)^{2}\right),
\end{aligned}
$$

where we have introduced $\sigma_{i j}=(i-j) /|i-j|$. We notice that

$$
V^{(1)}=-\frac{Q^{2}}{2 a^{2}} \sum_{i} \sum_{j \neq i} \sigma_{i j} \frac{q_{i}-q_{j}}{(i-j)^{2}}=0
$$

as one can easily verify by using the definition of $\sigma_{i j}$. This is satisfied also in the ion chain in presence of an axial trapping potential, since $V^{(1)}=0$ determines the equilibrium positions. 


\section{POTENTIAL TERMS FOR THE NORMAL MODES OF THE LINEAR CHAIN}

Here we report the third and fourth order terms of the potential, decomposed into the modes $\Theta_{k}=\Theta_{k}^{(+)}-i \Theta_{k}^{(-)}$, $\Psi_{k}^{\sigma}=\Psi_{k}^{\sigma(+)}-i \Psi_{k}^{\sigma(-)}$. The third order term takes the form

$$
\begin{aligned}
V^{(3)}= & \sum_{k_{1}+k_{2}+k_{3}=0} B\left(k_{1}, k_{2}, k_{3}\right) \\
& \times\left[\Theta_{k_{1}}\left(3 \sum_{\sigma=y, z} \Psi_{k_{2}}^{\sigma} \Psi_{k_{3}}^{\sigma}-2 \Theta_{k_{2}} \Theta_{k_{3}}\right)\right.
\end{aligned}
$$

where the sum runs over positive and negative values of $k_{j}$ and

$$
B\left(k_{1}, k_{2}, k_{3}\right)=-i \sqrt{\frac{2}{N}} \frac{Q^{2}}{a^{4}} \sum_{m>0} \frac{1}{m^{4}} \prod_{p=1}^{3} \sin \frac{k_{p} m a}{2}(.46)
$$

Term (45) is real, as it is visible by using the decomposition into even and odd modes. In particular, it has odd parity, coupling either three odd modes or two odd modes with an even one.

The quartic term reads

$$
\begin{aligned}
V^{(4)} & =\sum_{k_{1}+k_{2}+k_{3}+k_{4}=0} A\left(k_{1}, k_{2}, k_{3}, k_{4}\right) \\
& \times\left[\frac{3}{8} \sum_{\sigma, \sigma^{\prime}=y, z} \Psi_{k_{1}}^{\sigma} \Psi_{k_{2}}^{\sigma} \Psi_{k_{3}}^{\sigma^{\prime}} \Psi_{k_{4}}^{\sigma^{\prime}}+\Theta_{k_{1}} \Theta_{k_{2}} \Theta_{k_{3}} \Theta_{k_{4}}\right. \\
& \left.-3 \sum_{\sigma=y, z} \Psi_{k_{1}}^{\sigma} \Psi_{k_{2}}^{\sigma} \Theta_{k_{3}} \Theta_{k_{4}}\right]
\end{aligned}
$$

with

$$
A\left(k_{1}, k_{2}, k_{3}, k_{4}\right)=\frac{4}{N} \frac{Q^{2}}{a^{5}} \sum_{m>0} \frac{1}{m^{5}} \prod_{p=1}^{4} \sin \frac{k_{p} m a}{2}(48)
$$

This term is even, and it thus couples either four modes with the same parity, or two odd modes with two even ones.

[1] D. H. E. Dubin and T. M. O'Neil, Rev. Mod. Phys. 71, 87 (1999).

[2] F. Diedrich, E. Peik, J. M. Chen, W. Quint, and H. Walther, Phys. Rev. Lett. 59, 2931 (1987).

[3] R. Blumel, J. M. Chen, E. Peik, W. Quint, W. Schleich, Y. R. Shen, and H. Walther, Nature 334, 309 (1988).

[4] R. Blümel, C. Kappler, W. Quint, and H. Walther, Phys. Rev. A 40, 808 (1989).

[5] J. N. Tan, J. J. Bollinger, B. Jelenkovic, and D. J. Wineland, Phys. Rev. Lett. 75, 4198 (1995).
[6] W. M. Itano, J. J. Bollinger, J. N. Tan, B. Jelenkovic, X. P. Huang, and D. J. Wineland, Science 279, 686 (1998).

[7] L. Hornekær, N. Kjærgaard, A. M. Thommesen, and M. Drewsen, Phys. Rev. Lett. 86, 1994 (2001).

[8] N. Kjærgaard and M. Drewsen, Phys. Rev. Lett. 91, 095002 (2003).

[9] A. Mortensen, E. Nielsen, T. Matthey, and M. Drewsen, Phys. Rev. Lett. 96, 103001 (pages 4) (2006).

[10] J. I. Cirac and P. Zoller, Phys. Rev. Lett. 74, 4091 (1995).

[11] F. Schmidt-Kaler, H. Haffner, M. Riebe, S. Gulde, G. P. T. Lancaster, T. Deuschle, C. Becher, C. F. Roos, J. Eschner, and R. Blatt, Nature 422, 408 (2003).

[12] D. Leibfried, B. DeMarco, V. Meyer, D. Lucas, M. Barrett, J. Britton, W. M. Itano, B. Jelenkovic, C. Langer, T. Rosenband, et al., Nature 422, 412 (2003).

[13] C. Wunderlich, in Laser Physics at the Limit (Springer, Heidelberg, Berlin, New York, 2001), p. 26, URL arXiv.org:quant-ph/0111158.

[14] D. Porras and J. I. Cirac, Phys. Rev. Lett. 92, 207901 (2004).

[15] M. Pons, V. Ahufinger, C. Wunderlich, A. Sanpera, S. Braungardt, A. Sen(De), U. Sen, and M. Lewenstein, Phys. Rev. Lett. 98, 023003 (2007).

[16] J. Taylor and T. Calarco, arXiv:0706.1951 (2007).

[17] T. Dauxois, S. Ruffo, E. Arimondo, and M. Wilkens, Dynamics and Thermodynamics of Systems with LongRange Interactions, Lecture Notes in Physics 602 (Springer Verlag, New York, 2002).

[18] R. H. Baughman, S. O. Dantas, S. Stafstrom, A. A. Zakhidov, T. B. Mitchell, and D. H. E. Dubin, Science 288, 2018 (2000).

[19] G. Birkl, S. Kassner, and H. Walther, Nature 357, 310 (1992).

[20] I. Waki, S. Kassner, G. Birkl, and H. Walther, Phys. Rev. Lett. 68, 2007 (1992).

[21] T. B. Mitchell, J. J. Bollinger, W. M. Itano, and D. H. E. Dubin, Phys. Rev. Lett. 87, 183001 (2001).

[22] J. P. Schiffer, Phys. Rev. Lett. 70, 818 (1993).

[23] G. Piacente, I. V. Schweigert, J. J. Betouras, and F. M. Peeters, Phys. Rev. B 69, 045324 (pages 17) (2004).

[24] L. D. Landau and E. M. Lifshitz, Statistical Physics (Pergamon, Oxford, 1958).

[25] A. Steane, Appl. Phys. B 64, 623 (1997).

[26] D. F. V. James, Appl. Phys. B 66, 181 (1998).

[27] D. H. E. Dubin, Phys. Rev. E 55, 4017 (1997).

[28] G. Morigi and S. Fishman, Phys. Rev. Lett. 93, 170602 (2004).

[29] G. Morigi and S. Fishman, Phys. Rev. E 70, 066141 (2004).

[30] D. H. E. Dubin, Phys. Rev. Lett. 71, 2753 (1993).

[31] G. Morigi and S. Fishman, Journal of Physics B: Atomic, Molecular and Optical Physics 39, S221 (2006).

[32] M. G. Raizen, J. M. Gilligan, J. C. Bergquist, W. M. Itano, and D. J. Wineland, Phys. Rev. A 45, 6493 (1992).

[33] N. Ashcroft, Solid State Physics (Holt-Saunders Int. Eds., Tokyo, 1976).

[34] O. Biham, D. Mukamel, and S. Shtrikman, Phys. Rev. Lett. 56, 2191 (1986).

[35] D. Mukamel and M. B. Walker, Phys. Rev. Lett. 58, 2559 (1987).

[36] J. Yin and J. Javanainen, Phys. Rev. A 51, 3959 (1995). 